\title{
Linear Estimation of Location and Scale Parameters using Partial Maxima
}

\author{
Nickos Papadatos \\ Department of Mathematics, Section of Statistics and O.R., University of Athens, \\ Panepistemiopolis, 15784 Athens, Greece. e-mail: npapadat@math.uoa.gr
}

\begin{abstract}
Consider an i.i.d. sample $X_{1}^{*}, X_{2}^{*}, \ldots, X_{n}^{*}$ from a location-scale family, and assume that the only available observations consist of the partial maxima (or minima) sequence, $X_{1: 1}^{*}, X_{2: 2}^{*}, \ldots, X_{n: n}^{*}$, where $X_{j: j}^{*}=\max \left\{X_{1}^{*}, \ldots, X_{j}^{*}\right\}$. This kind of truncation appears in several circumstances, including best performances in athletics events. In the case of partial maxima, the form of the BLUEs (best linear unbiased estimators) is quite similar to the form of the well-known Lloyd's (1952, Least-squares estimation of location and scale parameters using order statistics, Biometrika, vol. 39, pp. 88-95) BLUEs, based on (the sufficient sample of) order statistics, but, in contrast to the classical case, their consistency is no longer obvious. The present paper is mainly concerned with the scale parameter, showing that the variance of the partial maxima BLUE is at most of order $O(1 / \log n)$, for a wide class of distributions.
\end{abstract}

Key words and phrases: Partial Maxima BLUEs; Location-scale family; Partial Maxima Spacings; S/BSW-type condition; NCP and NCS class; Log-concave distributions; Consistency for the scale estimator; Records.

\section{Introduction}

There are several situations where the ordered random sample,

$$
X_{1: n}^{*} \leqslant X_{2: n}^{*} \leqslant \cdots \leqslant X_{n: n}^{*},
$$

corresponding to the i.i.d. random sample, $X_{1}^{*}, X_{2}^{*}, \ldots, X_{n}^{*}$, is not fully reported, because the values of interest are the higher (or lower), up-to-the-present, record values based on the initial sample, i.e., the partial maxima (or minima) sequence

$$
X_{1: 1}^{*} \leqslant X_{2: 2}^{*} \leqslant \cdots \leqslant X_{n: n}^{*}
$$

where $X_{j: j}^{*}=\max \left\{X_{1}^{*}, \ldots, X_{j}^{*}\right\}$. A situation of this kind commonly appears in athletics, when only the best performances are recorded.

Through this article we assume that the i.i.d. data arise from a location-scale family,

$$
\left\{F\left(\left(\cdot-\theta_{1}\right) / \theta_{2}\right) ; \theta_{1} \in \mathbb{R}, \theta_{2}>0\right\},
$$


where the d.f. $F(\cdot)$ is free of parameters and has finite, non-zero variance (so that $F$ is non-degenerate), and we consider the partial maxima BLUE (best linear unbiased estimator) for both parameters $\theta_{1}$ and $\theta_{2}$. This consideration is along the lines of the classical Lloyd's (1952) BLUEs, the only difference being that the linear estimators are now based on the "insufficient sample" (1.2), rather than (1.1), and this fact implies a substantial reduction on the available information. Tryfos and Blackmore (1985) used this kind of data to predict future records in athletic events, Samaniego and Whitaker $(1986,1988)$ estimated the population characteristics, while Hofmann and Nagaraja (2003) investigated the amount of Fisher Information contained in such data; see also Arnold, Balakrisnan \& Nagaraja (1998, Section 5.9).

A natural question concerns the consistency of the resulting BLUEs, since too much lack of information presumably would result to inconsistency (see at the end of Section 6). Thus, our main focus is on conditions guaranteeing consistency, and the main result shows that this is indeed the case for the scale parameter BLUE from a wide class of distributions. Specifically, it is shown that the variance of the BLUE is at most of order $O(1 / \log n)$, when $F(x)$ has a log-concave density $f(x)$ and satisfies the Von Mises-type condition (5.11) or (6.1) (cf. Galambos (1978)) on the right endpoint of its support (Theorem 5.2, Corollary 6.1). The result is applicable to several commonly used distributions, like the Power distribution (Uniform), the Weibull (Exponential), the Pareto, the Negative Exponential, the Logistic, the Extreme Value (Gumbel) and the Normal (see section 6). A consistency result for the partial maxima BLUE of the location parameter would be desirable to be included here, but it seems that the proposed technique (based on partial maxima spacings, section 4) does not suffice for deriving it. Therefore, the consistency for the location parameter remains an open problem in general, and it is just highlighted by a particular application to the Uniform location-scale family (section 3).

The proof of the main result depends on the fact that, under mild conditions, the partial maxima spacings have non-positive correlation. The class of distributions having this property is called NCP (negative correlation for partial maxima spacings). It is shown here that any log-concave distribution with finite variance belongs to NCP (Theorem 4.2). In particular, if a distribution function has a density which is either log-concave or non-increasing then it is a member of NCP. For ordinary spacings, similar sufficient conditions were shown by Sarkadi (1985) and Bai, Sarkar \& Wang (1997) - see also David and Nagaraja (2003, pp. 187-188), Burkschat (2009), Theorem 3.5 - and will be referred as "S/BSW-type conditions".

In every experiment where the i.i.d. observations arise in a sequential manner, the partial maxima data describe the best performances in a natural way, as the experiment goes on, in contrast to the first $n$ record values, $R_{1}, R_{2}, \ldots, R_{n}$, which are obtained from an inverse sampling scheme - see, e.g., Berger and Gulati (2001). Due to the very rare appearance of records, in the latter case it is implicitly assumed that the sample size is, roughly, $e^{n}$. This has a similar effect in the partial maxima setup, since the number of different values are about $\log n$, for large sample size $n$. Clearly, the total amount of information in the partial maxima sample is the same as that given by the (few) record values augmented by record times. The essential 
difference of these models (records / partial maxima) in statistical applications is highlighted, e.g., in Tryfos and Blackmore (1985), Samaniego and Whitaker (1986, 1988), Smith (1988), Berger and Gulati (2001) and Hofmann and Nagaraja (2003) see also Arnold,Balakrishnan \& Nagaraja (1998, Chapter 5).

\section{Linear estimators based on partial maxima}

Consider the random sample $X_{1}^{*}, X_{2}^{*}, \ldots, X_{n}^{*}$ from $F\left(\left(x-\theta_{1}\right) / \theta_{2}\right)$ and the corresponding partial maxima sample $X_{1: 1}^{*} \leqslant X_{2: 2}^{*} \leqslant \cdots \leqslant X_{n: n}^{*}\left(\theta_{1} \in \mathbb{R}\right.$ is the location parameter and $\theta_{2}>0$ is the scale parameter; both parameters are unknown). Let also $X_{1}, X_{2}, \ldots, X_{n}$ and $X_{1: 1} \leqslant X_{2: 2} \leqslant \cdots \leqslant X_{n: n}$ be the corresponding samples from the completely specified d.f. $F(x)$, that generates the location-scale family. Since

$$
\left(X_{1: 1}^{*}, X_{2: 2}^{*}, \ldots, X_{n: n}^{*}\right)^{\prime} \stackrel{\mathrm{d}}{=}\left(\theta_{1}+\theta_{2} X_{1: 1}, \theta_{1}+\theta_{2} X_{2: 2}, \ldots, \theta_{1}+\theta_{2} X_{n: n}\right)^{\prime},
$$

a linear estimator based on partial maxima has the form

$$
L=\sum_{i=1}^{n} c_{i} X_{i: i}^{*} \stackrel{\mathrm{d}}{=} \theta_{1} \sum_{i=1}^{n} c_{i}+\theta_{2} \sum_{i=1}^{n} c_{i} X_{i: i}
$$

for some constants $c_{i}, i=1,2, \ldots, n$.

Let $\boldsymbol{X}=\left(X_{1: 1}, X_{2: 2}, \ldots, X_{n: n}\right)^{\prime}$ be the random vector of partial maxima from the known d.f. $F(x)$, and use the notation

$$
\boldsymbol{\mu}=\mathbb{E}[\boldsymbol{X}], \quad \boldsymbol{\Sigma}=\mathbb{D}[\boldsymbol{X}] \text { and } \boldsymbol{E}=\mathbb{E}\left[\boldsymbol{X} \boldsymbol{X}^{\prime}\right],
$$

where $\mathbb{D}[\boldsymbol{\xi}]$ denotes the dispersion matrix of any random vector $\boldsymbol{\xi}$. Clearly,

$$
\boldsymbol{\Sigma}=\boldsymbol{E}-\boldsymbol{\mu} \boldsymbol{\mu}^{\prime}, \quad \boldsymbol{\Sigma}>0, \quad \boldsymbol{E}>0
$$

The linear estimator $L$ is called BLUE for $\theta_{k}(k=1,2)$ if it is unbiased for $\theta_{k}$ and its variance is minimal, while it is called BLIE (best linear invariant estimator) for $\theta_{k}$ if it is invariant for $\theta_{k}$ and its mean squared error, $\operatorname{MSE}[L]=\mathbb{E}\left[L-\theta_{k}\right]^{2}$, is minimal. Here "invariance" is understood in the sense of location-scale invariance as it is defined, e.g., in Shao (2005, p. xix).

Using the above notation it is easy to verify the following formulae for the BLUEs and their variances. They are the partial maxima analogues of Lloyd's (1952) estimators and, in the case of partial minima, have been obtained by Tryfos and Blackmore (1985), using least squares. A proof is attached here for easy reference.

Proposition 2.1 The partial maxima BLUEs for $\theta_{1}$ and for $\theta_{2}$ are, respectively,

$$
L_{1}=-\frac{1}{\Delta} \boldsymbol{\mu}^{\prime} \boldsymbol{\Gamma} \boldsymbol{X}^{*} \text { and } L_{2}=\frac{1}{\Delta} \mathbf{1}^{\prime} \boldsymbol{\Gamma} \boldsymbol{X}^{*}
$$

where $\boldsymbol{X}^{*}=\left(X_{1: 1}^{*}, X_{2: 2}^{*}, \ldots, X_{n: n}^{*}\right)^{\prime}, \Delta=\left(\mathbf{1}^{\prime} \boldsymbol{\Sigma}^{-1} \mathbf{1}\right)\left(\boldsymbol{\mu}^{\prime} \boldsymbol{\Sigma}^{-1} \boldsymbol{\mu}\right)-\left(\mathbf{1}^{\prime} \boldsymbol{\Sigma}^{-1} \boldsymbol{\mu}\right)^{2}>0, \mathbf{1}=$ $(1,1, \ldots, 1)^{\prime} \in \mathbb{R}^{n}$ and $\boldsymbol{\Gamma}=\boldsymbol{\Sigma}^{-1}\left(\mathbf{1} \boldsymbol{\mu}^{\prime}-\boldsymbol{\mu} \mathbf{1}^{\prime}\right) \boldsymbol{\Sigma}^{-1}$. The corresponding variances are

$$
\operatorname{Var}\left[L_{1}\right]=\frac{1}{\Delta}\left(\boldsymbol{\mu}^{\prime} \boldsymbol{\Sigma}^{-1} \boldsymbol{\mu}\right) \theta_{2}^{2} \quad \text { and } \quad \operatorname{Var}\left[L_{2}\right]=\frac{1}{\Delta}\left(\mathbf{1}^{\prime} \boldsymbol{\Sigma}^{-1} \mathbf{1}\right) \theta_{2}^{2}
$$


Proof: Let $\boldsymbol{c}=\left(c_{1}, c_{2}, \ldots, c_{n}\right)^{\prime} \in \mathbb{R}^{n}$ and $L=\boldsymbol{c}^{\prime} \boldsymbol{X}^{*}$. Since $\mathbb{E}[L]=\left(\boldsymbol{c}^{\prime} \mathbf{1}\right) \theta_{1}+\left(\boldsymbol{c}^{\prime} \boldsymbol{\mu}\right) \theta_{2}$, $L$ is unbiased for $\theta_{1}$ iff $\boldsymbol{c}^{\prime} \mathbf{1}=1$ and $\boldsymbol{c}^{\prime} \boldsymbol{\mu}=0$, while it is unbiased for $\theta_{2}$ iff $\boldsymbol{c}^{\prime} \mathbf{1}=0$ and $\boldsymbol{c}^{\prime} \boldsymbol{\mu}=1$. Since $\operatorname{Var}[L]=\left(\boldsymbol{c}^{\prime} \boldsymbol{\Sigma} \boldsymbol{c}\right) \theta_{2}^{2}$, a simple minimization argument for $\boldsymbol{c}^{\prime} \boldsymbol{\Sigma} \boldsymbol{c}$ with respect to $\boldsymbol{c}$, using Lagrange multipliers, yields the expressions (2.2) and (2.3).

Similarly, one can derive the partial maxima version of Mann's (1969) best linear invariant estimators (BLIEs), as follows.

Proposition 2.2 The partial maxima BLIEs for $\theta_{1}$ and for $\theta_{2}$ are, respectively,

$$
T_{1}=\frac{\mathbf{1}^{\prime} \boldsymbol{E}^{-1} \boldsymbol{X}^{*}}{\mathbf{1}^{\prime} \boldsymbol{E}^{-1} \mathbf{1}} \text { and } T_{2}=\frac{\mathbf{1}^{\prime} \boldsymbol{G} \boldsymbol{X}^{*}}{\mathbf{1}^{\prime} \boldsymbol{E}^{-1} \mathbf{1}}
$$

where $\boldsymbol{X}^{*}$ and $\mathbf{1}$ are as in Proposition 2.1 and $\boldsymbol{G}=\boldsymbol{E}^{-1}\left(\mathbf{1} \boldsymbol{\mu}^{\prime}-\boldsymbol{\mu} \mathbf{1}^{\prime}\right) \boldsymbol{E}^{-1}$. The corresponding mean squared errors are

$$
\operatorname{MSE}\left[T_{1}\right]=\frac{\theta_{2}^{2}}{\mathbf{1}^{\prime} \boldsymbol{E}^{-1} \mathbf{1}} \text { and } \operatorname{MSE}\left[T_{2}\right]=\left(1-\frac{D}{\mathbf{1}^{\prime} \boldsymbol{E}^{-1} \mathbf{1}}\right) \theta_{2}^{2},
$$

where $D=\left(\mathbf{1}^{\prime} \boldsymbol{E}^{-1} \mathbf{1}\right)\left(\boldsymbol{\mu}^{\prime} \boldsymbol{E}^{-1} \boldsymbol{\mu}\right)-\left(\mathbf{1}^{\prime} \boldsymbol{E}^{-1} \boldsymbol{\mu}\right)^{2}>0$.

Proof: Let $L=L\left(\boldsymbol{X}^{*}\right)=\boldsymbol{c}^{\prime} \boldsymbol{X}^{*}$ be an arbitrary linear statistic. Since $L\left(b \boldsymbol{X}^{*}+a \mathbf{1}\right)=$ $a\left(\boldsymbol{c}^{\prime} \mathbf{1}\right)+b L\left(\boldsymbol{X}^{*}\right)$ for arbitrary $a \in \mathbb{R}$ and $b>0$, it follows that $L$ is invariant for $\theta_{1}$ iff $\boldsymbol{c}^{\prime} \mathbf{1}=1$ while it is invariant for $\theta_{2}$ iff $\boldsymbol{c}^{\prime} \mathbf{1}=0$. Both (2.4) and (2.5) now follow by a simple minimization argument, since in the first case we have to minimize the mean squared error $\mathbb{E}\left[L-\theta_{1}\right]^{2}=\left(\boldsymbol{c}^{\prime} \boldsymbol{E} \boldsymbol{c}\right) \theta_{2}^{2}$ under $\boldsymbol{c}^{\prime} \mathbf{1}=1$, while in the second one, we have to minimize the mean squared error $\mathbb{E}\left[L-\theta_{2}\right]^{2}=\left(\boldsymbol{c}^{\prime} \boldsymbol{E} \boldsymbol{c}-2 \boldsymbol{\mu}^{\prime} \boldsymbol{c}+1\right) \theta_{2}^{2}$ under $\boldsymbol{c}^{\prime} \mathbf{1}=0$.

The above formulae (2.2)-(2.5) are well-known for order statistics and records - see David (1981, Chapter 6), Arnold, Balakrishnan \& Nagaraja (1992, Chapter 7; 1998, Chapter 5), David and Nagaraja (2003, Chapter 8). In the present setup, however, the meaning of $\boldsymbol{X}^{*}, \boldsymbol{X}, \boldsymbol{\mu}, \boldsymbol{\Sigma}$ and $\boldsymbol{E}$ is completely different. In the case of order statistics, for example, the vector $\boldsymbol{\mu}$, which is the mean vector of the order statistics $\boldsymbol{X}=\left(X_{1: n}, X_{2: n}, \ldots, X_{n: n}\right)^{\prime}$ from the known distribution $F(x)$, depends on the sample size $n$, in the sense that the components of the vector $\boldsymbol{\mu}$ completely change with $n$. In the present case of partial maxima, the first $n$ entries of the vector $\boldsymbol{\mu}$, which is the mean vector of the partial maxima $\boldsymbol{X}=\left(X_{1: 1}, X_{2: 2}, \ldots, X_{n: n}\right)^{\prime}$ from the known distribution $F(x)$, remain constant for all sample sizes $n^{\prime}$ greater than or equal to $n$. Similar observations apply for the matrices $\boldsymbol{\Sigma}$ and $\boldsymbol{E}$. This fact seems to be quite helpful for the construction of tables giving the means, variances and covariances of partial maxima for samples up to a size $n$. It should be noted, however, that even when $F(x)$ is absolutely continuous with density $f(x)$ (as is usually the case for location-scale families), the joint distribution of $\left(X_{i: i}, X_{j: j}\right)$ has a singular part, since $\mathbb{P}\left[X_{i: i}=X_{j: j}\right]=i / j>0, i<j$. Nevertheless, there exist simple expectation and covariance formulae (Lemma 2.2). 
As in the order statistics setup, the actual application of formulae (2.2) and (2.4) requires closed forms for $\boldsymbol{\mu}$ and $\boldsymbol{\Sigma}$, and also to invert the $n \times n$ matrix $\boldsymbol{\Sigma}$. This can be done only for very particular distributions (see next section, where we apply the results to the Uniform distribution). Therefore, numerical methods should be applied in general. This, however, has a theoretical cost: It is not a trivial fact to verify consistency of the estimators, even in the classical case of order statistics. The main purpose of this article is in verifying consistency for the partial maxima BLUEs. Surprisingly, it seems that a solution of this problem is not well-known, at least to our knowledge, even for the classical BLUEs based on order statistics. However, even if the result of the following lemma is known, its proof has an independent interest, because it proposes alternative (to BLUEs) $n^{-1 / 2}$-consistent unbiased linear estimators and provides the intuition for the derivation of the main result of the present article.

Lemma 2.1 The classical BLUEs of $\theta_{1}$ and $\theta_{2}$, based on order statistics from a location-scale family, created by a distribution $F(x)$ with finite non-zero variance, are consistent. Moreover, their variance is at most of order $O(1 / n)$.

Proof: Let $\boldsymbol{X}^{*}=\left(X_{1: n}^{*}, X_{2: n}^{*}, \ldots, X_{n: n}^{*}\right)^{\prime}$ and $\boldsymbol{X}=\left(X_{1: n}, X_{2: n}, \ldots, X_{n: n}\right)^{\prime}$ be the ordered samples from $F\left(\left(x-\theta_{1}\right) / \theta_{2}\right)$ and $F(x)$, respectively, so that $\boldsymbol{X}^{*} \stackrel{\mathrm{d}}{=} \theta_{1} \mathbf{1}+\theta_{2} \boldsymbol{X}$. Also write $X_{1}^{*}, X_{2}^{*}, \ldots, X_{n}^{*}$ and $X_{1}, X_{2}, \ldots, X_{n}$ for the corresponding i.i.d. samples. We consider the linear estimators

$$
S_{1}=\bar{X}^{*}=\frac{1}{n} \sum_{i=1}^{n} X_{i}^{*} \stackrel{\mathrm{d}}{=} \theta_{1}+\theta_{2} \bar{X}
$$

and

$$
S_{2}=\frac{1}{n(n-1)} \sum_{i=1}^{n} \sum_{j=1}^{n}\left|X_{j}^{*}-X_{i}^{*}\right| \stackrel{\mathrm{d}}{=} \frac{\theta_{2}}{n(n-1)} \sum_{i=1}^{n} \sum_{j=1}^{n}\left|X_{j}-X_{i}\right|,
$$

i.e., $S_{1}$ is the sample mean and $S_{2}$ is a multiple of Gini's statistic. Observe that both $S_{1}$ and $S_{2}$ are linear estimators in order statistics. [In particular, $S_{2}$ can be written as $S_{2}=4(n(n-1))^{-1} \sum_{i=1}^{n}(i-(n+1) / 2) X_{i: n}^{*}$. Clearly, $\mathbb{E}\left(S_{1}\right)=\theta_{1}+\theta_{2} \mu_{0}$, $\mathbb{E}\left(S_{2}\right)=\theta_{2} \tau_{0}$, where $\mu_{0}$ is the mean, $\mathbb{E}\left(X_{1}\right)$, of the distribution $F(x)$ and $\tau_{0}$ is the positive finite parameter $\mathbb{E}\left|X_{1}-X_{2}\right|$. Since $F$ is known, both $\mu_{0} \in \mathbb{R}$ and $\tau_{0}>0$ are known constants, and we can construct the linear estimators $U_{1}=S_{1}-\left(\mu_{0} / \tau_{0}\right) S_{2}$ and $U_{2}=S_{2} / \tau_{0}$. Obviously, $\mathbb{E}\left(U_{k}\right)=\theta_{k}, k=1,2$, and both $U_{1}, U_{2}$ are linear estimators of the form $T_{n}=(1 / n) \sum_{i=1}^{n} \delta(i, n) X_{i: n}^{*}$, with $|\delta(i, n)|$ uniformly bounded for all $i$ and $n$. If $\sigma_{0}^{2}$ is the (assumed finite) variance of $F(x)$, it follows that

$$
\begin{aligned}
\operatorname{Var}\left[T_{n}\right] & \leqslant \frac{1}{n^{2}} \sum_{i=1}^{n} \sum_{j=1}^{n}|\delta(i, n)||\delta(j, n)| \operatorname{Cov}\left(X_{i: n}^{*}, X_{j: n}^{*}\right) \\
& \leqslant \frac{1}{n^{2}}\left(\max _{1 \leqslant i \leqslant n}|\delta(i, n)|\right)^{2} \operatorname{Var}\left(X_{1: n}^{*}+X_{2: n}^{*}+\cdots+X_{n: n}^{*}\right) \\
& =\frac{1}{n}\left(\max _{1 \leqslant i \leqslant n}|\delta(i, n)|\right)^{2} \theta_{2}^{2} \sigma_{0}^{2}=O\left(n^{-1}\right) \rightarrow 0, \quad \text { as } n \rightarrow \infty
\end{aligned}
$$


showing that $\operatorname{Var}\left(U_{k}\right) \rightarrow 0$, and thus $U_{k}$ is consistent for $\theta_{k}, k=1$, 2. Since $L_{k}$ has minimum variance among all linear unbiased estimators, it follows that $\operatorname{Var}\left(L_{k}\right) \leqslant$ $\operatorname{Var}\left(U_{k}\right) \leqslant O(1 / n)$, and the result follows.

The above lemma implies that the mean squared error of the BLIEs, based on order statistics, is at most of order $O(1 / n)$, since they have smaller mean squared error than the BLUEs, and thus they are also consistent. More important is the fact that, with the technique used in Lemma 2.1, one can avoid all computations involving means, variances and covariances of order statistics, and it does not need to invert any matrix, in order to prove consistency (and in order to obtain $O\left(n^{-1}\right)$-consistent estimators). Arguments of similar kind will be applied in section 5, when the problem of consistency for the partial maxima BLUE of $\theta_{2}$ will be taken under consideration.

We now turn in the partial maxima case. Since actual application of partial maxima BLUEs and BLIEs requires the computation of the first two moments of $\boldsymbol{X}=$ $\left(X_{1: 1}, X_{2: 2}, \ldots, X_{n: n}\right)^{\prime}$ in terms of the completely specified d.f. $F(x)$, the following formulae are to be mentioned here (cf. Jones and Balakrishnan (2002)).

Lemma 2.2 Let $X_{1: 1} \leqslant X_{2: 2} \leqslant \cdots \leqslant X_{n: n}$ be the partial maxima sequence based on an arbitrary d.f. $F(x)$.

(i) For $i \leqslant j$, the joint d.f. of $\left(X_{i: i}, X_{j: j}\right)$ is

$$
F_{X_{i: i}, X_{j: j}}(x, y)= \begin{cases}F^{j}(y) & \text { if } x \geqslant y \\ F^{i}(x) F^{j-i}(y) & \text { if } x \leqslant y\end{cases}
$$

(ii) If F has finite first moment, then

$$
\mu_{i}=\mathbb{E}\left[X_{i: i}\right]=\int_{0}^{\infty}\left(1-F^{i}(x)\right) d x-\int_{-\infty}^{0} F^{i}(x) d x
$$

is finite for all $i$.

(iii) If F has finite second moment, then

$$
\sigma_{i j}=\operatorname{Cov}\left[X_{i: i}, X_{j: j}\right]=\iint_{-\infty<x<y<\infty} F^{i}(x)\left(F^{j-i}(x)+F^{j-i}(y)\right)\left(1-F^{i}(y)\right) d y d x
$$

is finite and non-negative for all $i \leqslant j$. In particular,

$$
\sigma_{i i}=\sigma_{i}^{2}=\operatorname{Var}\left[X_{i: i}\right]=2 \iint_{-\infty<x<y<\infty} F^{i}(x)\left(1-F^{i}(y)\right) d y d x .
$$

Proof: (i) is trivial and (ii) is well-known. (iii) follows from Hoeffding's identity

$$
\operatorname{Cov}[X, Y]=\int_{-\infty}^{\infty} \int_{-\infty}^{\infty}\left(F_{X, Y}(x, y)-F_{X}(x) F_{Y}(y)\right) d y d x
$$

(see Hoeffding (1940), Lehmann (1966), Jones and Balakrishnan (2002), among others), applied to $(X, Y)=\left(X_{i: i}, X_{j: j}\right)$ with joint d.f. given by (2.6) and marginals $F^{i}(x)$ and $F^{j}(y)$. 
Formulae (2.7)-(2.9) enable the computation of means, variances and covariances of partial maxima, even in the case where the distribution $F$ does not have a density. Tryfos and Blackmore (1985) obtained an expression for the covariance of partial minima involving means and covariances of order statistics from lower sample sizes.

\section{A tractable case: the Uniform location-scale fam-I ily}

Let $X_{1}^{*}, X_{2}^{*}, \ldots, X_{n}^{*} \sim U\left(\theta_{1}, \theta_{1}+\theta_{2}\right)$, so that $\left(X_{1: 1}^{*}, X_{2: 2}^{*}, \ldots, X_{n: n}^{*}\right)^{\prime} \stackrel{\mathrm{d}}{=} \theta_{1} \mathbf{1}+\theta_{2} \boldsymbol{X}$, where $\boldsymbol{X}=\left(X_{1: 1}, X_{2: 2}, \ldots, X_{n: n}\right)^{\prime}$ is the partial maxima sample from the standard Uniform distribution. Simple calculations, using (2.7)-(2.9), show that the mean vector $\boldsymbol{\mu}=\left(\mu_{i}\right)$ and the dispersion matrix $\boldsymbol{\Sigma}=\left(\sigma_{i j}\right)$ of $\boldsymbol{X}$ are given by (see also Tryfos and Blackmore (1985), eq. (3.1))

$$
\mu_{i}=\frac{i}{i+1} \text { and } \sigma_{i j}=\frac{i}{(i+1)(j+1)(j+2)} \text { for } 1 \leqslant i \leqslant j \leqslant n .
$$

Therefore, $\boldsymbol{\Sigma}$ is a patterned matrix of the form $\sigma_{i j}=a_{i} b_{j}$ for $i \leqslant j$, and thus, its inverse is tridiagonal; see Graybill (1969, Chapter 8), Arnold, Balakrishnan \& Nagaraja (1992, Lemma 7.5.1). Specifically,

$$
\boldsymbol{\Sigma}^{-1}=\left(\begin{array}{cccccc}
\gamma_{1} & -\delta_{1} & 0 & \ldots & 0 & 0 \\
-\delta_{1} & \gamma_{2} & -\delta_{2} & \ldots & 0 & 0 \\
0 & -\delta_{2} & \gamma_{3} & \ldots & 0 & 0 \\
\vdots & \vdots & \vdots & \vdots & \vdots & \vdots \\
0 & 0 & 0 & \ldots & \gamma_{n-1} & -\delta_{n-1} \\
0 & 0 & 0 & \ldots & -\delta_{n-1} & \gamma_{n}
\end{array}\right)
$$

where

$$
\begin{gathered}
\gamma_{i}=\frac{4(i+1)^{3}(i+2)^{2}}{(2 i+1)(2 i+3)}, \quad \delta_{i}=\frac{(i+1)(i+2)^{2}(i+3)}{2 i+3}, \quad i=1,2, \ldots, n-1, \\
\text { and } \gamma_{n}=\frac{(n+1)^{2}(n+2)^{2}}{2 n+1} .
\end{gathered}
$$

Setting $a(n)=\mathbf{1}^{\prime} \boldsymbol{\Sigma}^{-1} \mathbf{1}, b(n)=(\mathbf{1}-\boldsymbol{\mu})^{\prime} \boldsymbol{\Sigma}^{-1}(\mathbf{1}-\boldsymbol{\mu})$ and $c(n)=(\mathbf{1}-\boldsymbol{\mu})^{\prime} \boldsymbol{\Sigma}^{-1} \mathbf{1}$, we get

$$
\begin{aligned}
& a(n)=\frac{(n+1)^{2}(n+2)^{2}}{2 n+1}-2 \sum_{i=1}^{n-1} \frac{(i+1)(i+2)^{2}(3 i+1)}{(2 i+1)(2 i+3)}=n^{2}+o\left(n^{2}\right), \\
& b(n)=\frac{(n+2)^{2}}{2 n+1}-2 \sum_{i=1}^{n-1} \frac{(i-1)(i+2)}{(2 i+1)(2 i+3)}=\frac{1}{2} \log n+o(\log n), \\
& c(n)=\frac{(n+1)(n+2)^{2}}{2 n+1}-\sum_{i=1}^{n-1} \frac{(i+2)\left(4 i^{2}+7 i+1\right)}{(2 i+1)(2 i+3)}=n+o(n) .
\end{aligned}
$$


Applying (2.3) we obtain

$$
\begin{aligned}
& \operatorname{Var}\left[L_{1}\right]=\frac{a(n)+b(n)-2 c(n)}{a(n) b(n)-c^{2}(n)} \theta_{2}^{2}=\left(\frac{2}{\log n}+o\left(\frac{1}{\log n}\right)\right) \theta_{2}^{2}, \text { and } \\
& \operatorname{Var}\left[L_{2}\right]=\frac{a(n)}{a(n) b(n)-c^{2}(n)} \theta_{2}^{2}=\left(\frac{2}{\log n}+o\left(\frac{1}{\log n}\right)\right) \theta_{2}^{2} .
\end{aligned}
$$

The preceding computation shows that, for the Uniform location-scale family, the partial maxima BLUEs are consistent for both the location and the scale parameters, since their variance goes to zero with the speed of $2 / \log n$. This fact, as expected, contradicts the behavior of the ordinary order statistics BLUEs, where the speed of convergence is of order $n^{-2}$ for the variance of both Lloyd's estimators. However, the comparison is quite unfair here, since Lloyd's estimators are based on the complete sufficient statistic $\left(X_{1: n}^{*}, X_{n: n}^{*}\right)$, and thus the variance of order statistics BLUE is minimal among all unbiased estimators.

On the other hand we should emphasize that, under the same model, the BLUEs (and the BLIEs) based solely on the first $n$ upper records are not even consistent. In fact, the variance of both BLUEs converges to $\theta_{2}^{2} / 3$, and the MSE of both BLIEs approaches $\theta_{2}^{2} / 4$, as $n \rightarrow \infty$; see Arnold, Balakrishnan \& Nagaraja (1998, Examples 5.3.7 and 5.4.3).

\section{Scale estimation and partial maxima spacings}

In the classical order statistics setup, Balakrishnan and Papadatos (2002) observed that the computation of BLUE (and BLIE) of the scale parameter is simplified considerably if one uses spacings instead of order statistics - cf. Sarkadi (1985). Their observation applies here too, and simplifies the form of the partial maxima BLUE (and BLIE).

Specifically, define the partial maxima spacings as $Z_{i}^{*}=X_{i+1: i+1}^{*}-X_{i: i}^{*} \geqslant 0$ and $Z_{i}=X_{i+1: i+1}-X_{i: i} \geqslant 0$, for $i=1,2, \ldots, n-1$, and let $Z^{*}=\left(Z_{1}^{*}, Z_{2}^{*}, \ldots, Z_{n-1}^{*}\right)^{\prime}$

and $\boldsymbol{Z}=\left(Z_{1}, Z_{2}, \ldots, Z_{n-1}\right)^{\prime}$. Clearly, $\boldsymbol{Z}^{*} \stackrel{\mathrm{d}}{=} \theta_{2} \boldsymbol{Z}$, and any unbiased (or even invariant) linear estimator of $\theta_{2}$ based on the partial maxima sample, $L=\boldsymbol{c}^{\prime} \boldsymbol{X}^{*}$, should necessarily satisfy $\sum_{i=1}^{n} c_{i}=0$ (see the proofs of Propositions 2.1 and 2.2). Therefore, $L$ can be expressed as a linear function on $Z_{i}^{*}$ 's, $L=\boldsymbol{b}^{\prime} \boldsymbol{Z}^{*}$, where now $\boldsymbol{b}=\left(b_{1}, b_{2}, \ldots, b_{n-1}\right)^{\prime} \in \mathbb{R}^{n-1}$. Consider the mean vector $\boldsymbol{m}=\mathbb{E}[\boldsymbol{Z}]$, the dispersion matrix $\boldsymbol{S}=\mathbb{D}[\boldsymbol{Z}]$, and the second moment matrix $\boldsymbol{D}=\mathbb{E}\left[\boldsymbol{Z} \boldsymbol{Z}^{\prime}\right]$ of $\boldsymbol{Z}$. Clearly, $\boldsymbol{S}=\boldsymbol{D}-\boldsymbol{m} \boldsymbol{m}^{\prime}, \boldsymbol{S}>0, \boldsymbol{D}>0$, and the vector $\boldsymbol{m}$ and the matrices $\boldsymbol{S}$ and $\boldsymbol{D}$ are of order $n-1$. Using exactly the same arguments as in Balakrishnan and Papadatos (2002), it is easy to verify the following.

Proposition 4.1 The partial maxima BLUE of $\theta_{2}$, given in Proposition 2.1, has the alternative form

$$
L_{2}=\frac{\boldsymbol{m}^{\prime} \boldsymbol{S}^{-1} \boldsymbol{Z}^{*}}{\boldsymbol{m}^{\prime} \boldsymbol{S}^{-1} \boldsymbol{m}}, \quad \text { with } \quad \operatorname{Var}\left[L_{2}\right]=\frac{\theta_{2}^{2}}{\boldsymbol{m}^{\prime} \boldsymbol{S}^{-1} \boldsymbol{m}},
$$


while the corresponding BLIE, given in Proposition 2.2, has the alternative form

$$
T_{2}=\boldsymbol{m}^{\prime} \boldsymbol{D}^{-1} \boldsymbol{Z}^{*}, \quad \text { with } \quad \operatorname{MSE}\left[T_{2}\right]=\left(1-\boldsymbol{m}^{\prime} \boldsymbol{D}^{-1} \boldsymbol{m}\right) \theta_{2}^{2} .
$$

It should be noted that, in general, the non-negativity of the BLUE of $\theta_{2}$ does not follow automatically, even for order statistics. In the order statistics setup, this problem was posed by Arnold, Balakrishnan \& Nagaraja (1992), and the best known result, till now, is the one given by Bai, Sarkar \& Wang (1997) and Sarkadi (1985). Even after the slight improvement, given by Balakrishnan and Papadatos (2002) and by Burkschat (2009), the general case remains unsolved. The same question (of nonnegativity of the BLUE) arises in the partial maxima setup, and the following theorem provides a partial positive answer. We omit the proof, since it again follows by a straightforward application of the arguments given in Balakrishnan and Papadatos (2002).

Theorem 4.1 (i) There exists a constant $a=a_{n}(F), 0<a<1$, depending only on the sample size $n$ and the d.f. $F(x)$ (i.e., $a$ is free of the parameters $\theta_{1}$ and $\left.\theta_{2}\right)$, such that $T_{2}=a L_{2}$. This constant is given by $a=\boldsymbol{m}^{\prime} \boldsymbol{D}^{-1} \boldsymbol{m}=\boldsymbol{m}^{\prime} \boldsymbol{S}^{-1} \boldsymbol{m} /\left(1+\boldsymbol{m}^{\prime} \boldsymbol{S}^{-1} \boldsymbol{m}\right)$. (ii) If either $n=2$ or the (free of parameters) d.f. $F(x)$ is such that

$$
\operatorname{Cov}\left[Z_{i}, Z_{j}\right] \leqslant 0 \quad \text { for all } i \neq j, \quad i, j=1, \ldots, n-1,
$$

then the partial maxima BLUE (and BLIE) of $\theta_{2}$ is non-negative.

Note that, as in order statistics, the non-negativity of $L_{2}$ is equivalent to the fact that the vector $\boldsymbol{S}^{-1} \boldsymbol{m}$ (or, equivalently, the vector $\boldsymbol{D}^{-1} \boldsymbol{m}$ ) has non-negative entries; see Balakrishnan and Papadatos (2002) and Sarkadi (1985).

Since it is important to know whether (4.3) holds, in the sequel we shall make use of the following definition.

Definition 4.1 A d.f. $F(x)$ with finite second moment (or the corresponding density $f(x)$, if exists)

(i) belongs to the class NCS (negatively correlated spacings) if its order statistics have negatively correlated spacings for all sample sizes $n \geqslant 2$.

(ii) belongs to the class NCP if it has negatively correlated partial maxima spacings, i.e., if (4.3) holds for all $n \geqslant 2$.

An important result by Bai, Sarkar \& Wang (1997) states that a log-concave density $f(x)$ with finite variance belongs to NCS - cf. Sarkadi (1985). We call this sufficient condition as the S/BSW-condition (for ordinary spacings). Burkschat (2009, Theorem 3.5) showed an extended S/BSW-condition, under which the log-concavity of both $F$ and $1-F$ suffice for the NCS class. Due to the existence of simple formulae like (4.1) and (4.2), the NCS and NCP classes provide useful tools in verifying consistency for the scale estimator, as well as, non-negativity. Our purpose is to prove an S/BSW-type condition for partial maxima (see Theorem 4.2, Corollary 4.1, below). To this end, we first state Lemma 4.1, that will be used in the sequel.

Only through the rest of the present section, we shall use the notation $Y_{k}=$ $\max \left\{X_{1}, \ldots, X_{k}\right\}$, for any integer $k \geqslant 1$. 
Lemma 4.1 Fix two integers $i, j$, with $1 \leqslant i<j$, and suppose that the i.i.d. r.v.'s $X_{1}, X_{2}, \ldots$ have a common d.f. $F(x)$. Let I(expression) denoting the indicator function taking the value 1 , if the expression holds true, and 0 otherwise.

(i) The conditional d.f. of $Y_{j+1}$ given $Y_{j}$ is

$$
\mathbb{P}\left[Y_{j+1} \leqslant y \mid Y_{j}\right]=\left\{\begin{array}{cl}
0, & \text { if } y<Y_{j} \\
F(y), & \text { if } y \geqslant Y_{j}
\end{array}=F(y) I\left(y \geqslant Y_{j}\right), \quad y \in \mathbb{R} .\right.
$$

If, in addition, $i+1<j$, then the following property (which is an immediate consequence of the Markovian character of the extremal process) holds:

$$
\mathbb{P}\left[Y_{j+1} \leqslant y \mid Y_{i+1}, Y_{j}\right]=\mathbb{P}\left[Y_{j+1} \leqslant y \mid Y_{j}\right], \quad y \in \mathbb{R}
$$

(ii) The conditional d.f. of $Y_{i}$ given $Y_{i+1}$ is

$$
\begin{aligned}
\mathbb{P}\left[Y_{i} \leqslant x \mid Y_{i+1}\right] & =\left\{\begin{array}{cl}
\frac{F^{i}(x)}{\sum_{j=0}^{i} F^{j}\left(Y_{i+1}\right) F^{i-j}\left(Y_{i+1}-\right)}, & \text { if } x<Y_{i+1} \\
1, & \text { if } x \geqslant Y_{i+1}
\end{array}\right. \\
& =I\left(x \geqslant Y_{i+1}\right)+I\left(x<Y_{i+1}\right) \frac{F^{i}(x)}{\sum_{j=0}^{i} F^{j}\left(Y_{i+1}\right) F^{i-j}\left(Y_{i+1}-\right)}, \quad x \in \mathbb{R} .
\end{aligned}
$$

If, in addition, $i+1<j$, then the following property (which is again an immediate consequence of the Markovian character of the extremal process) holds:

$$
\mathbb{P}\left[Y_{i} \leqslant x \mid Y_{i+1}, Y_{j}\right]=\mathbb{P}\left[Y_{i} \leqslant x \mid Y_{i+1}\right], \quad x \in \mathbb{R}
$$

(iii) Given $\left(Y_{i+1}, Y_{j}\right)$, the random variables $Y_{i}$ and $Y_{j+1}$ are independent.

We omit the proof since the assertions are simple by-products of the Markovian character of the process $\left\{Y_{k}, k \geqslant 1\right\}$, which can be embedded in a continuous time extremal process $\{Y(t), t>0\}$; see Resnick (1987, Chapter 4). We merely note that a version of the Radon-Nikodym derivative of $F^{i+1}$ w.r.t. $F$ is given by

$$
h_{i+1}(x)=\frac{d F^{i+1}(x)}{d F(x)}=\sum_{j=0}^{i} F^{j}(x) F^{i-j}(x-), \quad x \in \mathbb{R}
$$

which is equal to $(i+1) F^{i}(x)$ only if $x$ is a continuity point of $F$. To see this, it suffices to verify the identity

$$
\int_{B} d F^{i+1}(x)=\int_{B} h_{i+1}(x) d F(x) \text { for all Borel sets } B \subseteq \mathbb{R}
$$

Now (4.5) is proved as follows:

$$
\int_{B} d F^{i+1}=\mathbb{P}\left(Y_{i+1} \in B\right)
$$




$$
\begin{aligned}
& =\sum_{j=1}^{i+1} \mathbb{P}\left[Y_{i+1} \in B, \sum_{k=1}^{i+1} I\left(X_{k}=Y_{i+1}\right)=j\right] \\
& =\sum_{j=1}^{i+1} \sum_{1 \leqslant k_{1}<\cdots<k_{j} \leqslant i+1} \mathbb{P}\left[X_{k_{1}}=\cdots=X_{k_{j}} \in B,\right. \\
& \left.X_{s}<X_{k_{1}} \text { for } s \notin\left\{k_{1}, \ldots, k_{j}\right\}\right] \\
& =\sum_{j=1}^{i+1}\left(\begin{array}{c}
i+1 \\
j
\end{array}\right) \mathbb{P}\left[X_{1}=\cdots=X_{j} \in B, X_{j+1}<X_{1}, \ldots, X_{i+1}<X_{1}\right] \\
& =\sum_{j=1}^{i+1}\left(\begin{array}{c}
i+1 \\
j
\end{array}\right) \int_{B} \mathbb{E}\left[\left(\prod_{k=2}^{j} I\left(X_{k}=x\right)\right)\left(\prod_{k=j+1}^{i+1} I\left(X_{k}<x\right)\right)\right] d F(x) \\
& =\int_{B}\left(\sum_{j=1}^{i+1}\left(\begin{array}{c}
i+1 \\
j
\end{array}\right)(F(x)-F(x-))^{j-1} F^{i+1-j}(x-)\right) d F(x) \\
& =\int_{B} h_{i+1}(x) d F(x),
\end{aligned}
$$

where we used the identity $\sum_{j=1}^{i+1}\left(\begin{array}{c}i+1 \\ j\end{array}\right)(b-a)^{j-1} a^{i+1-j}=\sum_{j=0}^{i} b^{j} a^{i-j}, a \leqslant b$.

We can now show the main result of this section, which presents an S/BSW-type condition for the partial maxima spacings.

Theorem 4.2 Assume that the d.f. $F(x)$, with finite second moment, is a log-concave distribution (in the sense that $\log F(x)$ is a concave function in $J$, where $J=\{x \in$ $\mathbb{R}: 0<F(x)<1\})$, and has not an atom at its right end-point, $\omega(F)=\inf \{x \in \mathbb{R}:$ $F(x)=1\}$. Then, $F(x)$ belongs to the class $\mathrm{NCP}$, i.e., (4.3) holds for all $n \geqslant 2$.

Proof: For arbitrary r.v.'s $X \geqslant x_{0}>-\infty$ and $Y \leqslant y_{0}<+\infty$, with respective d.f.'s $F_{X}, F_{Y}$, we have

$$
\mathbb{E}[X]=x_{0}+\int_{x_{0}}^{\infty}\left(1-F_{X}(t)\right) d t \text { and } \quad \mathbb{E}[Y]=y_{0}-\int_{-\infty}^{y_{0}} F_{Y}(t) d t
$$

(cf. Papadatos (2001), Jones and Balakrishnan (2002)). Assume that $i<j$. By Lemma 4.1(i) and (4.6) applied to $F_{X}=F_{Y_{j+1} \mid Y_{j}}$, it follows that

$$
\mathbb{E}\left[Y_{j+1} \mid Y_{i+1}, Y_{j}\right]=\mathbb{E}\left[Y_{j+1} \mid Y_{j}\right]=Y_{j}+\int_{Y_{j}}^{\infty}(1-F(t)) d t, \quad \text { w.p. } 1 .
$$

Similarly, by Lemma 4.1(ii) and (4.6) applied to $F_{Y}=F_{Y_{i} \mid Y_{i+1}}$, we conclude that

$$
\mathbb{E}\left[Y_{i} \mid Y_{i+1}, Y_{j}\right]=\mathbb{E}\left[Y_{i} \mid Y_{i+1}\right]=Y_{i+1}-\frac{1}{h_{i+1}\left(Y_{i+1}\right)} \int_{-\infty}^{Y_{i+1}} F^{i}(t) d t, \quad \text { w.p. 1, }
$$

where $h_{i+1}$ is given by (4.4). Note that $F$ is continuous on $J$, since it is log-concave there, and thus, $h_{i+1}(x)=(i+1) F^{i}(x)$ for $x \in J$. If $\omega(F)$ is finite, $F(x)$ is also 
continuous at $x=\omega(F)$, by assumption. On the other hand, if $\alpha(F)=\inf \{x: F(x)>$ $0\}$ is finite, $F$ can be discontinuous at $x=\alpha(F)$, but in this case, $h_{i+1}(\alpha(F))=$ $F^{i}(\alpha(F))>0$; see (4.4). Thus, in all cases, $h_{i+1}\left(Y_{i+1}\right)>0$ w.p. 1.

By conditional independence of $Y_{i}$ and $Y_{j+1}$ (Lemma 4.1(iii)), we have

$$
\begin{aligned}
\operatorname{Cov}\left(Z_{i}, Z_{j} \mid Y_{i+1}, Y_{j}\right) & =\operatorname{Cov}\left(Y_{i+1}-Y_{i}, Y_{j+1}-Y_{j} \mid Y_{i+1}, Y_{j}\right) \\
& =-\operatorname{Cov}\left(Y_{i}, Y_{j+1} \mid Y_{i+1}, Y_{j}\right)=0, \quad \text { w.p. 1, }
\end{aligned}
$$

so that $\mathbb{E}\left[\operatorname{Cov}\left(Z_{i}, Z_{j} \mid Y_{i+1}, Y_{j}\right)\right]=0$, and thus,

$$
\begin{aligned}
\operatorname{Cov}\left[Z_{i}, Z_{j}\right] & =\operatorname{Cov}\left[\mathbb{E}\left(Z_{i} \mid Y_{i+1}, Y_{j}\right), \mathbb{E}\left(Z_{j} \mid Y_{i+1}, Y_{j}\right)\right]+\mathbb{E}\left[\operatorname{Cov}\left(Z_{i}, Z_{j} \mid Y_{i+1}, Y_{j}\right)\right] \\
& =\operatorname{Cov}\left[\mathbb{E}\left(Y_{i+1}-Y_{i} \mid Y_{i+1}, Y_{j}\right), \mathbb{E}\left(Y_{j+1}-Y_{j} \mid Y_{i+1}, Y_{j}\right)\right] \\
& =\operatorname{Cov}\left[Y_{i+1}-\mathbb{E}\left(Y_{i} \mid Y_{i+1}, Y_{j}\right), \mathbb{E}\left(Y_{j+1} \mid Y_{i+1}, Y_{j}\right)-Y_{j}\right] \\
& =\operatorname{Cov}\left[g\left(Y_{i+1}\right), h\left(Y_{j}\right)\right],
\end{aligned}
$$

where

$$
g(x)=\left\{\begin{array}{ll}
\frac{1}{(i+1) F^{i}(x)} \int_{-\infty}^{x} F^{i}(t) d t, & x>\alpha(F), \\
0, & \text { otherwise, }
\end{array} \quad h(x)=\int_{x}^{\infty}(1-F(t)) d t .\right.
$$

Obviously, $h(x)$ is non-increasing. On the other hand, $g(x)$ is non-decreasing in $\mathbb{R}$. This can be shown as follows. First observe that $g(\alpha(F))=0$ if $\alpha(F)$ is finite, while $g(x)>0$ for $x>\alpha(F)$. Next observe that $g$ is finite and continuous at $x=\omega(F)$ if $\omega(F)$ is finite, as follows by the assumed continuity of $F$ at $x=\omega(F)$ and the fact that $F$ has finite variance. Finally, observe that $F^{i}(x)$, a product of log-concave functions, is also log-concave in $J$. Therefore, for arbitrary $y \in J$, the function $d(x)=F^{i}(x) / \int_{-\infty}^{y} F^{i}(t) d t, x \in(-\infty, y) \cap J$, is a probability density, and thus, it is a log-concave density with support $(-\infty, y) \cap J$. By Prèkopa (1973) or Dasgupta and Sarkar (1982) it follows that the corresponding distribution function, $D(x)=\int_{-\infty}^{x} d(t) d t=\int_{-\infty}^{x} F^{i}(t) d t / \int_{-\infty}^{y} F^{i}(t) d t, x \in(-\infty, y) \cap J$, is a log-concave distribution, and since $y$ is arbitrary, $H(x)=\int_{-\infty}^{x} F^{i}(t) d t$ is a log-concave function, for $x \in J$. Since $F$ is continuous in $J$, this is equivalent to the fact that the function

$$
\frac{H^{\prime}(x)}{H(x)}=\frac{F^{i}(x)}{\int_{-\infty}^{x} F^{i}(t) d t}, \quad x \in J
$$

is non-increasing, so that $g(x)=H(x) /\left((i+1) H^{\prime}(x)\right)$ is non-decreasing in $J$.

The desired result follows from (4.7), because the r.v.'s $Y_{i+1}$ and $Y_{j}$ are positively quadrant dependent (PQD - Lehmann (1966)), since it is readily verified that $F_{Y_{i+1}, Y_{j}}(x, y) \geqslant F_{Y_{i+1}}(x) F_{Y_{j}}(y)$ for all $x$ and $y$ (Lemma 2.2(i)). This completes the proof.

The restriction $F(x) \rightarrow 1$ as $x \rightarrow \omega(F)$ cannot be removed from the theorem. Indeed, the function

$$
F(x)= \begin{cases}0 & x \leqslant 0 \\ x / 4, & 0 \leqslant x<1 \\ 1, & x \geqslant 1\end{cases}
$$


is a log-concave distribution in $J=(\alpha(F), \omega(F))=(0,1)$, for which $\operatorname{Cov}\left[Z_{1}, Z_{2}\right]=$ $\frac{59}{184320}>0$. The function $g$, used in the proof, is given by

$$
g(x)= \begin{cases}\max \left\{0, \frac{x}{(i+1)^{2}}\right\}, & x<1, \\ \frac{x-1}{i+1}+\frac{1}{(i+1)^{2} 4^{i}}, & x \geqslant 1,\end{cases}
$$

and it is not monotonic.

Since the family of densities with log-concave distributions contains both families of log-concave and non-increasing densities (see, e.g., Prèkopa (1973), Dasgupta and Sarkar (1982), Sengupta and Nanda (1999), Bagnoli and Bergstrom (2005)), the following corollary is an immediate consequence of Theorems 4.1 and 4.2.

Corollary 4.1 Assume that $F(x)$ has finite second moment.

(i) If $F(x)$ is a log-concave d.f. (in particular, if $F(x)$ has either a log-concave or a non-increasing (in its interval support) density $f(x)$ ), then the partial maxima BLUE and the partial maxima BLIE of $\theta_{2}$ are non-negative.

(ii) If $F(x)$ has either a log-concave or a non-increasing (in its interval support) density $f(x)$ then it belongs to the NCP class.

Sometimes it is asserted that "the distribution of a log-convex density is logconcave" (see, e.g., Sengupta and Nanda (1999), Proposition 1(e)), but this is not correct in its full generality, even if the corresponding r.v. $X$ is non-negative. For example, let $Y \sim$ Weibull with shape parameter $1 / 2$, and set $X \stackrel{\mathrm{d}}{=} Y \mid Y<1$. Then $X$ has density $f$ and d.f. $F$ given by

$$
f(x)=\frac{\exp (-\sqrt{1-x})}{2\left(1-e^{-1}\right) \sqrt{1-x}}, \quad F(x)=\frac{\exp (-\sqrt{1-x})-e^{-1}}{1-e^{-1}}, \quad 0<x<1,
$$

and it is easily checked that $\log f$ is convex in $J=(0,1)$, while $F$ is not log-concave in $J$. However, we point out that if $\sup J=+\infty$ then any log-convex density, supported on $J$, has to be non-increasing in $J$ and, therefore, its distribution is log-concave in $J$. Examples of log-convex distributions having a log-convex density are given by Bagnoli and Bergstrom (2005).

\section{Consistent estimation of the scale parameter}

Through this section we always assume that $F(x)$, the d.f. that generates the locationscale family, is non-degenerate and has finite second moment. The main purpose is to verify consistency for $L_{2}$, applying the results of section 4 . To this end, we firstly state and prove a simple lemma that goes through the lines of Lemma 2.1. Due to the obvious fact that $\operatorname{MSE}\left[T_{2}\right] \leqslant \operatorname{Var}\left[L_{2}\right]$, all the results of the present section apply

also to the BLIE of $\theta_{2}$. 
Lemma 5.1 If $F(x)$ belongs to the NCP class then

(i)

$$
\operatorname{Var}\left[L_{2}\right] \leqslant \frac{\theta_{2}^{2}}{\sum_{k=1}^{n-1} m_{k}^{2} / s_{k}^{2}},
$$

where $m_{k}=\mathbb{E}\left[Z_{k}\right]$ is the $k$-th component of the vector $\boldsymbol{m}$ and $s_{k}^{2}=s_{k k}=\operatorname{Var}\left[Z_{k}\right]$ is the $k$-th diagonal entry of the matrix $\boldsymbol{S}$.

(ii) The partial maxima BLUE, $L_{2}$, is consistent if the series

$$
\sum_{k=1}^{\infty} \frac{m_{k}^{2}}{s_{k}^{2}}=+\infty
$$

Proof: Observe that part (ii) is an immediate consequence of part (i), due to the fact that, in contrast to the order statistics setup, $m_{k}$ and $s_{k}^{2}$ do not depend on the sample size $n$. Regarding (i), consider the linear unbiased estimator

$$
U_{2}=\frac{1}{c_{n}} \sum_{k=1}^{n-1} \frac{m_{k}}{s_{k}^{2}} Z_{k}^{*} \stackrel{\mathrm{d}}{=} \frac{\theta_{2}}{c_{n}} \sum_{k=1}^{n-1} \frac{m_{k}}{s_{k}^{2}} Z_{k},
$$

where $c_{n}=\sum_{k=1}^{n-1} m_{k}^{2} / s_{k}^{2}$. Since $F(x)$ belongs to NCP and the weights of $U_{2}$ are positive, it follows that the variance of $U_{2}$, which is greater than or equal to the variance of $L_{2}$, is bounded by the RHS of (5.1); this completes the proof.

The proof of the following theorem is now immediate.

Theorem 5.1 If $F(x)$ belongs to the NCP class and if there exists a finite constant $C$ and a positive integer $k_{0}$ such that

$$
\frac{\mathbb{E}\left[Z_{k}^{2}\right]}{k \mathbb{E}^{2}\left[Z_{k}\right]} \leqslant C, \text { for all } k \geqslant k_{0},
$$

then

$$
\operatorname{Var}\left[L_{2}\right] \leqslant O\left(\frac{1}{\log n}\right), \text { as } n \rightarrow \infty .
$$

Proof: Since for $k \geqslant k_{0}$,

$$
\frac{m_{k}^{2}}{s_{k}^{2}}=\frac{m_{k}^{2}}{\mathbb{E}\left[Z_{k}^{2}\right]-m_{k}^{2}}=\frac{1}{\frac{\mathbb{E}\left[Z_{k}^{2}\right]}{\mathbb{E}^{2}\left[Z_{k}\right]}-1} \geqslant \frac{1}{C k-1},
$$

the result follows by (5.1).

Thus, for proving consistency of order $1 / \log n$ into NCP class it is sufficient to verify (5.3) and, therefore, we shall investigate the quantities $m_{k}=\mathbb{E}\left[Z_{k}\right]$ and 
$\mathbb{E}\left[Z_{k}^{2}\right]=s_{k}^{2}+m_{k}^{2}$. A simple application of Lemma 2.2, observing that $m_{k}=\mu_{k+1}-\mu_{k}$ and $s_{k}^{2}=\sigma_{k+1}^{2}-2 \sigma_{k, k+1}+\sigma_{k}^{2}$, shows that

$$
\begin{aligned}
\mathbb{E}\left[Z_{k}\right] & =\int_{-\infty}^{\infty} F^{k}(x)(1-F(x)) d x \\
\mathbb{E}^{2}\left[Z_{k}\right] & =2 \iint_{-\infty<x<y<\infty} F^{k}(x)(1-F(x)) F^{k}(y)(1-F(y)) d y d x, \\
\mathbb{E}\left[Z_{k}^{2}\right] & =2 \iint_{-\infty<x<y<\infty} F^{k}(x)(1-F(y)) d y d x .
\end{aligned}
$$

Therefore, all the quantities of interest can be expressed as integrals in terms of the (completely arbitrary) d.f. $F(x)$ (cf. Jones and Balakrishnan (2002)).

For the proof of the main result we finally need the following lemma and its corollary.

Lemma 5.2 (i) For any $t>-1$,

$$
\lim _{k \rightarrow \infty} k^{1+t} \int_{0}^{1} u^{k}(1-u)^{t} d u=\Gamma(1+t)>0 .
$$

(ii) For any $t$ with $0 \leqslant t<1$ and any $a>0$, there exist positive constants $C_{1}, C_{2}$, and a positive integer $k_{0}$ such that

$$
0<C_{1}<k^{1+t}(\log k)^{a} \int_{0}^{1} \frac{u^{k}(1-u)^{t}}{L^{a}(u)} d u<C_{2}<\infty, \text { for all } k \geqslant k_{0},
$$

where $L(u)=-\log (1-u)$.

Proof: Part (i) follows by Stirling's formula. For part (ii), with the substitution $u=1-e^{-x}$, we write the integral in (5.9) as

$$
\frac{1}{k+1} \int_{0}^{\infty}(k+1)\left(1-e^{-x}\right)^{k} e^{-x} \frac{\exp (-t x)}{x^{a}} d x=\frac{1}{k+1} \mathbb{E}\left[\frac{\exp (-t T)}{T^{a}}\right],
$$

where $T$ has the same distribution as the maximum of $k+1$ i.i.d. standard exponential r.v.'s. It is well-known that $\mathbb{E}[T]=1^{-1}+2^{-1}+\cdots+(k+1)^{-1}$. Since the second derivative of the function $x \rightarrow e^{-t x} / x^{a}$ is $x^{-a-2} e^{-t x}\left(a+(a+t x)^{2}\right)$, which is positive for $x>0$, this function is convex, so by Jensen's inequality we conclude that

$$
\begin{aligned}
k^{1+t}(\log k)^{a} \int_{0}^{1} \frac{u^{k}(1-u)^{t}}{(L(u))^{a}} d u \geqslant & \frac{k}{k+1}\left(\frac{\log k}{1+1 / 2+\cdots+1 /(k+1)}\right)^{a} \\
& \times \exp \left[-t\left(1+\frac{1}{2}+\cdots+\frac{1}{k+1}-\log k\right)\right],
\end{aligned}
$$

and the RHS remains positive as $k \rightarrow \infty$, since it converges to $e^{-\gamma t}$, where $\gamma=$ $.5772 \ldots$ is Euler's constant. This proves the lower bound in (5.9). Regarding the 
upper bound, observe that the function $g(u)=(1-u)^{t} / L^{a}(u), u \in(0,1)$, has second derivative

$$
g^{\prime \prime}(u)=\frac{-(1-u)^{t-2}}{L^{a+2}(u)}\left[t(1-t) L^{2}(u)+a(1-2 t) L(u)-a(a+1)\right], \quad 0<u<1,
$$

and since $0 \leqslant t<1, a>0$ and $L(u) \rightarrow+\infty$ as $u \rightarrow 1-$, it follows that there exists a constant $b \in(0,1)$ such that $g(u)$ is concave in $(b, 1)$. Split now the integral in $(5.9)$ in two parts,

$$
I_{k}=I_{k}^{(1)}(b)+I_{k}^{(2)}(b)=\int_{0}^{b} \frac{u^{k}(1-u)^{t}}{L^{a}(u)} d u+\int_{b}^{1} \frac{u^{k}(1-u)^{t}}{L^{a}(u)} d u,
$$

and observe that for any fixed $s>0$ and any fixed $b \in(0,1)$,

$$
k^{s} I_{k}^{(1)}(b) \leqslant k^{s} b^{k-a} \int_{0}^{b} \frac{u^{a}(1-u)^{t}}{L^{a}(u)} d u \leqslant k^{s} b^{k-a} \int_{0}^{1} \frac{u^{a}(1-u)^{t}}{L^{a}(u)} d u \rightarrow 0, \quad \text { as } k \rightarrow \infty,
$$

because the last integral is finite and independent of $k$. Therefore, $k^{1+t}(\log k)^{a} I_{k}$ is bounded above if $k^{1+t}(\log k)^{a} I_{k}^{(2)}(b)$ is bounded above for some $b<1$. Choose $b$ close enough to 1 so that $g(u)$ is concave in $(b, 1)$. By Jensen's inequality and the fact that $1-b^{k+1}<1$ we conclude that

$$
I_{k}^{(2)}(b)=\frac{1-b^{k+1}}{k+1} \int_{b}^{1} f_{k}(u) g(u) d u=\frac{1-b^{k+1}}{k+1} \mathbb{E}[g(V)] \leqslant \frac{1}{k+1} g[\mathbb{E}(V)],
$$

where $V$ is an r.v. with density $f_{k}(u)=(k+1) u^{k} /\left(1-b^{k+1}\right)$, for $u \in(b, 1)$. Since $\mathbb{E}(V)=((k+1) /(k+2))\left(1-b^{k+2}\right) /\left(1-b^{k+1}\right)>(k+1) /(k+2)$, and $g$ is positive and decreasing (its first derivative is $g^{\prime}(u)=-(1-u)^{t-1} L^{-a-1}(u)(t L(u)+a)<0$, $0<u<1$ ), it follows from the above inequality that

$$
\begin{array}{r}
k^{1+t}(\log k)^{a} I_{k}^{(2)}(b) \leqslant \frac{k^{1+t}(\log k)^{a}}{k+1} g[\mathbb{E}(V)] \leqslant \frac{k^{1+t}(\log k)^{a}}{k+1} g\left(\frac{k+1}{k+2}\right) \\
=\frac{k}{k+1}\left(\frac{\log k}{\log (k+2)}\right)^{a}\left(\frac{k}{k+2}\right)^{t} \leqslant 1
\end{array}
$$

This shows that $k^{1+t}(\log k)^{a} I_{k}^{(2)}(b)$ is bounded above, and thus, $k^{1+t}(\log k)^{a} I_{k}$ is bounded above, as it was to be shown. The proof is complete.

Corollary 5.1 (i) Under the assumptions of Lemma 5.2(i), for any $b \in[0,1)$ there exist positive constants $A_{1}, A_{2}$ and a positive integer $k_{0}$ such that

$$
0<A_{1}<k^{1+t} \int_{b}^{1} u^{k}(1-u)^{t} d u<A_{2}<+\infty, \text { for all } k \geqslant k_{0} .
$$

(ii) Under the assumptions of Lemma 5.2(ii), for any $b \in[0,1)$ there exist positive constants $A_{3}, A_{4}$ and a positive integer $k_{0}$ such that

$$
0<A_{3}<k^{1+t}(\log k)^{a} \int_{b}^{1} \frac{u^{k}(1-u)^{t}}{L^{a}(u)} d u<A_{4}<\infty, \text { for all } k \geqslant k_{0} .
$$


Proof: The proof follows from Lemma 5.2 in a trivial way, since the corresponding integrals over $[0, b]$ are bounded above by a multiple of $b^{k-a}$, of the form $A b^{k-a}$, with $A<+\infty$ being independent of $k$.

We can now state and prove our main result:

Theorem 5.2 Assume that $F(x)$ lies in $\mathrm{NCP}$, and let $\omega=\omega(F)$ be the upper endpoint of the support of $F$, i.e., $\omega=\inf \{x \in \mathbb{R}: F(x)=1\}$, where $\omega=+\infty$ if $F(x)<1$ for all $x$. Suppose that $\lim _{x \rightarrow \omega-} F(x)=1$, and that $F(x)$ is differentiable in a left neighborhood $(M, \omega)$ of $\omega$, with derivative $f(x)=F^{\prime}(x)$ for $x \in(M, \omega)$. For $\delta \in \mathbb{R}$ and $\gamma \in \mathbb{R}$, define the (generalized hazard rate) function

$$
L(x)=L(x ; \delta, \gamma ; F)=\frac{f(x)}{(1-F(x))^{\gamma}(-\log (1-F(x)))^{\delta}}, \quad x \in(M, \omega),
$$

and set

$$
L_{*}=L_{*}(\delta, \gamma ; F)=\liminf _{x \rightarrow \omega-} L(x ; \delta, \gamma, F), \quad L^{*}=L^{*}(\delta, \gamma ; F)=\limsup _{x \rightarrow \omega-} L(x ; \delta, \gamma, F) .
$$

If either (i) for some $\gamma<3 / 2$ and $\delta=0$, or (ii) for some $\delta>0$ and some $\gamma$ with $1 / 2<\gamma \leqslant 1$,

$$
0<L_{*}(\delta, \gamma ; F) \leqslant L^{*}(\delta, \gamma ; F)<+\infty,
$$

then the partial maxima BLUE $L_{2}$ (given by (2.2) or (4.1)) of the scale parameter $\theta_{2}$ is consistent and, moreover, $\operatorname{Var}\left[L_{2}\right] \leqslant O(1 / \log n)$.

Proof: First observe that for large enough $x<\omega$, (5.11) implies that $f(x)>$ $\left(L_{*} / 2\right)(1-F(x))^{\gamma}(-\log (1-F(x)))^{\delta}>0$, so that $F(x)$ is eventually strictly increasing and continuous. Moreover, the derivative $f(x)$ is necessarily finite since $f(x)<2 L^{*}(1-F(x))^{\gamma}(-\log (1-F(x)))^{\delta}$. The assumption $\lim _{x \rightarrow \omega-} F(x)=1$ now shows that $F^{-1}(u)$ is uniquely defined in a left neighborhood of 1 , that $F\left(F^{-1}(u)\right)=u$ for $u$ close to 1 , and that $\lim _{u \rightarrow 1-} F^{-1}(u)=\omega$. This, in turn, implies that $F^{-1}(u)$ is differentiable for $u$ close to 1 , with (finite) derivative $\left(F^{-1}(u)\right)^{\prime}=1 / f\left(F^{-1}(u)\right)>0$. In view of Theorem 5.1, it suffices to verify (5.3), and thus we seek for an upper bound on $\mathbb{E}\left[Z_{k}^{2}\right]$ and for a lower bound on $\mathbb{E}\left[Z_{k}\right]$. Clearly, (5.3) will be deduced if we shall verify that, under (i), there exist finite constants $C_{3}>0, C_{4}>0$ such that

$$
k^{3-2 \gamma} \mathbb{E}\left[Z_{k}^{2}\right] \leqslant C_{3} \quad \text { and } \quad k^{2-\gamma} \mathbb{E}\left[Z_{k}\right] \geqslant C_{4},
$$

for all large enough $k$. Similarly, (5.3) will be verified if we show that, under (ii), there exist finite constants $C_{5}>0$ and $C_{6}>0$ such that

$$
k^{3-2 \gamma}(\log k)^{2 \delta} \mathbb{E}\left[Z_{k}^{2}\right] \leqslant C_{5} \quad \text { and } \quad k^{2-\gamma}(\log k)^{\delta} \mathbb{E}\left[Z_{k}\right] \geqslant C_{6},
$$

for all large enough $k$. Since the integrands in the integral expressions (5.5)-(5.7) vanish if $x$ or $y$ lies outside the set $\{x \in \mathbb{R}: 0<F(x)<1\}$, we have the equivalent 
expressions

$$
\begin{aligned}
\mathbb{E}\left[Z_{k}\right] & =\int_{\alpha}^{\omega} F^{k}(x)(1-F(x)) d x, \\
\mathbb{E}\left[Z_{k}^{2}\right] & =2 \iint_{\alpha<x<y<\omega} F^{k}(x)(1-F(y)) d y d x,
\end{aligned}
$$

where $\alpha$ (resp., $\omega$ ) is the lower (resp., the upper) end-point of the support of $F$. Obviously, for any fixed $M$ with $\alpha<M<\omega$ and any fixed $s>0$, we have, as in the proof of Lemma 5.2(ii), that

$$
\begin{array}{r}
\lim _{k \rightarrow \infty} k^{s} \int_{\alpha}^{M} F^{k}(x)(1-F(x)) d x=0, \\
\lim _{k \rightarrow \infty} k^{s} \int_{\alpha}^{M} \int_{x}^{\omega} F^{k}(x)(1-F(y)) d y d x=0,
\end{array}
$$

because $F(M)<1$ and both integrals (5.14), (5.15) are finite for $k=1$, by the assumption that the variance is finite $((5.15)$ with $k=1$ just equals to the variance of $F$; see also (2.9) with $i=1)$. Therefore, in order to verify (5.12) and (5.13) for large enough $k$, it is sufficient to replace $\mathbb{E}\left[Z_{k}\right]$ and $\mathbb{E}\left[Z_{k}^{2}\right]$, in both formulae (5.12), (5.13), by the integrals $\int_{M}^{\omega} F^{k}(x)(1-F(x)) d x$ and $\int_{M}^{\omega} \int_{x}^{\omega} F^{k}(x)(1-F(y)) d y d x$, respectively, for an arbitrary (fixed) $M \in(\alpha, \omega)$. Fix now $M \in(\alpha, \omega)$ so large that $f(x)=F^{\prime}(x)$ exists and it is finite and strictly positive for all $x \in(M, \omega)$, and make the transformation $F(x)=u$ in the first integral, and the transformation $(F(x), F(y))=(u, v)$ in the second one. Both transformations are now one-to-one and continuous, because both $F$ and $F^{-1}$ are differentiable in their respective intervals $(M, \omega)$ and $(F(M), 1)$, and their derivatives are finite and positive. Since $F^{-1}(u) \rightarrow \omega$ as $u \rightarrow 1-$, it is easily seen that (5.12) will be concluded if it can be shown that for some fixed $b<1$ (which can be chosen arbitrarily close to 1 ),

$$
\begin{gathered}
k^{3-2 \gamma} \int_{b}^{1} \frac{u^{k}}{f\left(F^{-1}(u)\right)}\left(\int_{u}^{1} \frac{1-v}{f\left(F^{-1}(v)\right)} d v\right) d u \leqslant C_{3} \quad \text { and } \\
k^{2-\gamma} \int_{b}^{1} \frac{u^{k}(1-u)}{f\left(F^{-1}(u)\right)} d u \geqslant C_{4},
\end{gathered}
$$

holds for all large enough $k$. Similarly, (5.13) will be deduced if it will be proved that for some fixed $b<1$ (which can be chosen arbitrarily close to 1 ),

$$
\begin{gathered}
k^{3-2 \gamma}(\log k)^{2 \delta} \int_{b}^{1} \frac{u^{k}}{f\left(F^{-1}(u)\right)}\left(\int_{u}^{1} \frac{1-v}{f\left(F^{-1}(v)\right)} d v\right) d u \leqslant C_{5} \quad \text { and } \\
k^{2-\gamma}(\log k)^{\delta} \int_{b}^{1} \frac{u^{k}(1-u)}{f\left(F^{-1}(u)\right)} d u \geqslant C_{6},
\end{gathered}
$$

holds for all large enough $k$. The rest of the proof is thus concentrated on showing (5.16) and (5.17) (resp., ((5.18) and (5.19)), under the assumption (i) (resp., under the assumption (ii)). 
Assume first that (5.11) holds under (i). Fix now $b<1$ so large that

$$
\frac{L_{*}}{2}(1-F(x))^{\gamma}<f(x)<2 L^{*}(1-F(x))^{\gamma}, \text { for all } x \in\left(F^{-1}(b), \omega\right) ;
$$

equivalently,

$$
\frac{1}{2 L^{*}}<\frac{(1-u)^{\gamma}}{f\left(F^{-1}(u)\right)}<\frac{2}{L_{*}}, \quad \text { for all } u \in(b, 1) .
$$

Due to (5.20), the inner integral in (5.16) is

$$
\int_{u}^{1} \frac{1-v}{f\left(F^{-1}(v)\right)} d v=\int_{u}^{1}(1-v)^{1-\gamma} \frac{(1-v)^{\gamma}}{f\left(F^{-1}(v)\right)} d v \leqslant \frac{2(1-u)^{2-\gamma}}{(2-\gamma) L_{*}}
$$

By Corollary 5.1(i) applied for $t=2-2 \gamma>-1$, the LHS of (5.16) is less than or equal to

$$
\frac{2 k^{3-2 \gamma}}{(2-\gamma) L_{*}} \int_{b}^{1} u^{k}(1-u)^{2-2 \gamma} \frac{(1-u)^{\gamma}}{f\left(F^{-1}(u)\right)} d u \leqslant \frac{4 k^{3-2 \gamma}}{(2-\gamma) L_{*}^{2}} \int_{b}^{1} u^{k}(1-u)^{2-2 \gamma} d u \leqslant C_{3},
$$

for all $k \geqslant k_{0}$, with $C_{3}=4 A_{2} L_{*}^{-2}(2-\gamma)^{-1}<\infty$, showing (5.16). Similarly, using the lower bound in (5.20), the integral in (5.17) is

$$
\int_{b}^{1} \frac{u^{k}(1-u)}{f\left(F^{-1}(u)\right)} d u=\int_{b}^{1} u^{k}(1-u)^{1-\gamma} \frac{(1-u)^{\gamma}}{f\left(F^{-1}(u)\right)} d u \geqslant \frac{1}{2 L^{*}} \int_{b}^{1} u^{k}(1-u)^{1-\gamma} d u,
$$

so that, by Corollary 5.1(i) applied for $t=1-\gamma>-1$, the LHS of (5.17) is greater than or equal to

$$
\frac{k^{2-\gamma}}{2 L^{*}} \int_{b}^{1} u^{k}(1-u)^{1-\gamma} d u \geqslant \frac{A_{1}}{2 L^{*}}>0, \quad \text { for all } k \geqslant k_{0},
$$

showing (5.17).

Assume now that (5.11) is satisfied under (ii). As in part (i), choose a large enough $b<1$ so that

$$
\frac{1}{2 L^{*}}<\frac{(1-u)^{\gamma} L^{\delta}(u)}{f\left(F^{-1}(u)\right)}<\frac{2}{L_{*}}, \quad \text { for all } u \in(b, 1),
$$

where $L(u)=-\log (1-u)$. Due to (5.21), the inner integral in (5.18) is

$$
\int_{u}^{1} \frac{(1-v)^{1-\gamma}}{L^{\delta}(v)} \frac{(1-v)^{\gamma} L^{\delta}(v)}{f\left(F^{-1}(v)\right)} d v \leqslant \frac{2}{L_{*}} \int_{u}^{1} \frac{(1-v)^{1-\gamma}}{L^{\delta}(v)} d v \leqslant \frac{2(1-u)^{2-\gamma}}{L_{*} L^{\delta}(u)},
$$

because $(1-u)^{1-\gamma} / L^{\delta}(u)$ is decreasing (see the proof of Lemma 5.2(ii)). By Corollary 5.1(ii) applied for $t=2-2 \gamma \in[0,1)$ and $a=2 \delta>0$, the double integral in (5.18) is less than or equal to

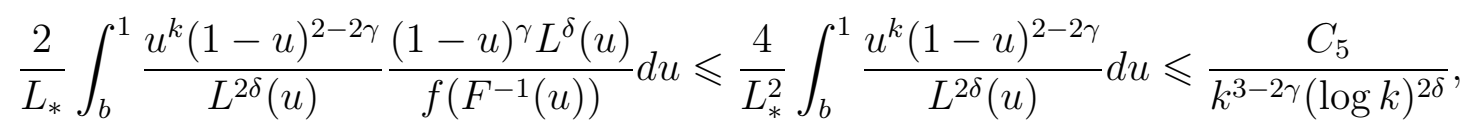


for all $k \geqslant k_{0}$, with $C_{5}=4 A_{4} L_{*}^{-2}<\infty$, showing (5.18). Similarly, using the lower bound in (5.21), the integral in (5.19) is

$$
\int_{b}^{1} \frac{u^{k}(1-u)^{1-\gamma}}{L^{\delta}(u)} \frac{(1-u)^{\gamma} L^{\delta}(u)}{f\left(F^{-1}(u)\right)} d u \geqslant \frac{1}{2 L^{*}} \int_{b}^{1} \frac{u^{k}(1-u)^{1-\gamma}}{L^{\delta}(u)} d u
$$

and thus, by Corollary 5.1(ii) applied for $t=1-\gamma \in[0,1)$ and $a=\delta>0$, the LHS of (5.19) is greater than or equal to

$$
\frac{k^{2-\gamma}(\log k)^{\delta}}{2 L^{*}} \int_{b}^{1} \frac{u^{k}(1-u)^{1-\gamma}}{L^{\delta}(u)} d u \geqslant \frac{A_{3}}{2 L^{*}}>0, \quad \text { for all } k \geqslant k_{0},
$$

showing (5.19). This completes the proof.

Remark 5.1 Taking $L(u)=-\log (1-u)$, the limits $L_{*}$ and $L^{*}$ in $(5.11)$ can be rewritten as

$$
\begin{aligned}
& L_{*}(\delta, \gamma ; F)=\liminf _{u \rightarrow 1-} \frac{f\left(F^{-1}(u)\right)}{(1-u)^{\gamma} L^{\delta}(u)}=\left(\limsup _{u \rightarrow 1-}\left(F^{-1}(u)\right)^{\prime}(1-u)^{\gamma} L^{\delta}(u)\right)^{-1}, \\
& L^{*}(\delta, \gamma ; F)=\limsup _{u \rightarrow 1-} \frac{f\left(F^{-1}(u)\right)}{(1-u)^{\gamma} L^{\delta}(u)}=\left(\liminf _{u \rightarrow 1-}\left(F^{-1}(u)\right)^{\prime}(1-u)^{\gamma} L^{\delta}(u)\right)^{-1} .
\end{aligned}
$$

In the particular case where $F$ is absolutely continuous with a continuous density $f$ and interval support, the function $f\left(F^{-1}(u)\right)=1 /\left(F^{-1}(u)\right)^{\prime}$ is known as the densityquantile function (Parzen, (1979)), and plays a fundamental role in the theory of order statistics. Theorem 5.2 shows, in some sense, that the behavior of the densityquantile function at the upper end-point, $u=1$, specifies the variance behavior of the partial maxima BLUE for the scale parameter $\theta_{2}$. In fact, (5.11) (and (6.1), below) is a Von Mises-type condition (cf. Galambos (1978), $\S \S 2.7,2.11$ ).

Remark 5.2 It is obvious that condition $\lim _{x \rightarrow \omega-} F(x)=1$ is necessary for the consistency of BLUE (and BLIE). Indeed, the event that all partial maxima are equal to $\omega(F)$ has probability $p_{0}=F(\omega)-F(\omega-)$ (which is independent of $n$ ). Thus, a point mass at $x=\omega(F)$ implies that for all $n, \mathbb{P}\left(L_{2}=0\right) \geqslant p_{0}>0$. This situation is trivial. Non-trivial cases also exist, and we provide one at the end of next section.

\section{Examples and conclusions}

In most commonly used location-scale families, the following corollary suffices for concluding consistency of the BLUE (and the BLIE) of the scale parameter. Its proof follows by a straightforward combination of Corollary 4.1(ii) and Theorem 5.2.

Corollary 6.1 Suppose that $F$ is absolutely continuous with finite variance, and that its density $f$ is either log-concave or non-increasing in its interval support 
$J=(\alpha(F), \omega(F))=\{x \in \mathbb{R}: 0<F(x)<1\}$. If, either for some $\gamma<3 / 2$ and $\delta=0$, or for some $\delta>0$ and some $\gamma$ with $1 / 2<\gamma \leqslant 1$,

$$
\lim _{x \rightarrow \omega(F)-} \frac{f(x)}{(1-F(x))^{\gamma}(-\log (1-F(x)))^{\delta}}=L \in(0,+\infty),
$$

then the partial maxima BLUE of the scale parameter is consistent and, moreover, its variance is at most of order $O(1 / \log n)$.

Corollary 6.1 has immediate applications to several location-scale families. The following are some of them, where (6.1) can be verified easily. In all these families generated by the distributions mentioned below, the variance of the partial maxima BLUE $L_{2}$ (see (2.3) or (4.1)), and the mean squared error of the partial maxima BLIE $T_{2}$ (see (2.5) or (4.2)) of the scale parameter is at most of order $O(1 / \log n$ ), as the sample size $n \rightarrow \infty$.

1. Power distribution (Uniform). $F(x)=x^{\lambda}, f(x)=\lambda x^{\lambda-1}, 0<x<1(\lambda>0)$, and $\omega(F)=1$. The density is non-increasing for $\lambda \leqslant 1$ and log-concave for $\lambda \geqslant 1$. It is easily seen that (6.1) is satisfied for $\delta=\gamma=0$ (for $\lambda=1$ (Uniform) see section 3).

2. Logistic distribution. $F(x)=\left(1+e^{-x}\right)^{-1}, f(x)=e^{-x}\left(1+e^{-x}\right)^{-2}, x \in \mathbb{R}$, and $\omega(F)=+\infty$. The density is log-concave, and it is easily seen that (6.1) is satisfied for $\delta=0, \gamma=1$.

3. Pareto distribution. $F(x)=1-x^{-a}, f(x)=a x^{-a-1}, x>1(a>2$, so that the second moment is finite), and $\omega(F)=+\infty$. The density is decreasing, and it is easily seen that (6.1) is satisfied for $\delta=0, \gamma=1+1 / a$. Pareto case provides an example which lies in NCP and not in NCS class - see Bai, Sarkar \& Wang (1997).

4. Negative Exponential distribution. $F(x)=f(x)=e^{x}, x<0$, and $\omega(F)=0$. The density is log-concave and it is easily seen that (6.1) is satisfied for $\delta=\gamma=0$. This model is particularly important, because it corresponds to the partial minima model from the standard exponential distribution - see Samaniego and Whitaker (1986).

5. Weibull distribution (Exponential). $F(x)=1-e^{-x^{c}}, f(x)=c x^{c-1} \exp \left(-x^{c}\right)$, $x>0(c>0)$, and $\omega(F)=+\infty$. The density is non-increasing for $c \leqslant 1$ and logconcave for $c \geqslant 1$, and it is easily seen that (6.1) is satisfied for $\delta=1-1 / c, \gamma=1$. It should be noted that Theorem 5.2 does not apply for $c<1$, since $\delta<0$.

6. Gumbel (Extreme Value) distribution. $F(x)=\exp \left(-e^{-x}\right)=e^{x} f(x), x \in \mathbb{R}$, and $\omega(F)=+\infty$. The distribution is log-concave and (6.1) holds with $\gamma=1, \delta=0$ $(L=1)$. This model is particularly important for its applications in forecasting records, especially in athletic events - see Tryfos and Blackmore (1985).

7. Normal Distribution. $f(x)=\varphi(x)=\left(2 \pi e^{x^{2}}\right)^{-1 / 2}, F=\Phi, x \in \mathbb{R}$, and $\omega(F)=$ $+\infty$. The density is log-concave and Corollary 6.1 applies with $\delta=1 / 2$ and $\gamma=1$. Indeed,

$$
\lim _{+\infty} \frac{\varphi(x)}{(1-\Phi(x))(-\log (1-\Phi(x)))^{1 / 2}}=\lim _{+\infty} \frac{\varphi(x)}{x(1-\Phi(x))} \frac{x}{(-\log (1-\Phi(x)))^{1 / 2}},
$$


and it is easily seen that

$$
\lim _{+\infty} \frac{\varphi(x)}{x(1-\Phi(x))}=1, \quad \lim _{+\infty} \frac{x^{2}}{-\log (1-\Phi(x))}=2,
$$

so that $L=\sqrt{2}$.

In many cases of interest (especially in athletic events), best performances are presented as partial minima rather than maxima; see, e.g., Tryfos and Blackmore (1985). Obviously, the present theory applies also to the partial minima setup. The easiest way to convert the present results for the partial minima case is to consider the i.i.d. sample $-X_{1}, \ldots,-X_{n}$, arising from $F_{-X}(x)=1-F_{X}(-x-)$, and to observe that $\min \left\{X_{1}, \ldots, X_{i}\right\}=-\max \left\{-X_{1}, \ldots,-X_{i}\right\}, i=1, \ldots, n$. Thus, we just have to replace $F(x)$ by $1-F(-x-)$ in the corresponding formulae.

There are some related problems and questions that, at least to our point of view, seem to be quite interesting. One problem is to verify consistency for the partial maxima BLUE of the location parameter. Another problem concerns the complete characterizations of the NCP and NCS classes (see Definition 4.1), since we only know S/BSW-type sufficient conditions. Also, to prove or disprove the nonnegativity of the partial maxima BLUE for the scale parameter, outside the NCP class (as well as for the order statistics BLUE of the scale parameter outside the NCS class).

Some questions concern lower variance bounds for the partial maxima BLUEs. For example we showed in section 3 that the rate $O(1 / \log n)$ (which, by Theorem 5.2 , is just an upper bound for the variance of $L_{2}$ ) is the correct order for the variance of both estimators in the Uniform location-scale family. Is this the usual case? If it is so, then we could properly standardize the estimators, centering and multiplying them by $(\log n)^{1 / 2}$. This would result to limit theorems analogues to the corresponding ones for order statistics - e.g., Chernoff, Gastwirth \& Johns (1967); Stigler (1974); - or analogues to the corresponding ones of Pyke (1965), (1980), for partial maxima spacings instead of ordinary spacings. However, note that the Fisher-Information approach, in the particular case of the one-parameter (scale) family generated by the standard Exponential distribution, suggests a variance of about $3 \theta_{2}^{2} /(\log n)^{3}$ for the minimum variance unbiased estimator (based on partial maxima) - see Hoffman and Nagaraja (2003, eq. (15) on p. 186).

A final question concerns the construction of approximate BLUEs (for both location and scale) based on partial maxima, analogues to Gupta's (1952) simple linear estimators based on order statistics. Such a kind of approximations and/or limit theorems would be especially useful for practical purposes, since the computation of BLUE via its closed formula requires inverting an $n \times n$ matrix. This problem has been partially solved here: For the NCP class, the estimator $U_{2}$, given in the proof of Lemma 5.1, is consistent for $\theta_{2}$ (under the assumptions of Theorem 5.2) and can be computed by a simple formula if we merely know the means and variances of the partial maxima spacings.

Except of the trivial case given in Remark 5.2, above, there exist non-trivial examples where no consistent sequence of unbiased estimators exist for the scale 
parameter. To see this, we make use of the following result.

Theorem 6.1 (Hofmann and Nagaraja 2003, p. 183) Let $X_{1}^{*}, X_{2}^{*}, \ldots, X_{n}^{*}$ be an i.i.d. sample from the scale family with distribution function $F\left(x ; \theta_{2}\right)=F\left(x / \theta_{2}\right)\left(\theta_{2}>0\right.$ is the scale parameter) and density $f\left(x ; \theta_{2}\right)=f\left(x / \theta_{2}\right) / \theta_{2}$, where $f(x)$ is known, it has a continuous derivative $f^{\prime}$, and its support, $J(F)=\{x: f(x)>0\}$, is one of the intervals $(-\infty, \infty),(-\infty, 0)$ or $(0,+\infty)$.

(i) The Fisher Information contained in the partial maxima data $X_{1: 1}^{*} \leqslant X_{2: 2}^{*} \leqslant \cdots \leqslant$ $X_{n: n}^{*}$ is given by

$$
I_{n}^{\max }=\frac{1}{\theta_{2}^{2}} \sum_{k=1}^{n} \int_{J(F)} f(x) F^{k-1}(x)\left(1+\frac{x f^{\prime}(x)}{f(x)}+\frac{(k-1) x f(x)}{F(x)}\right)^{2} d x .
$$

(ii) The Fisher Information contained in the partial minima data $X_{1: 1}^{*} \geqslant X_{1: 2}^{*} \geqslant \cdots \geqslant$ $X_{1: n}^{*}$ is given by

$$
I_{n}^{\min }=\frac{1}{\theta_{2}^{2}} \sum_{k=1}^{n} \int_{J(F)} f(x)(1-F(x))^{k-1}\left(1+\frac{x f^{\prime}(x)}{f(x)}-\frac{(k-1) x f(x)}{1-F(x)}\right)^{2} d x .
$$

It is clear that for fixed $\theta_{2}>0, I_{n}^{\max }$ and $I_{n}^{\min }$ both increase with the sample size $n$. In particular, if $J(F)=(0, \infty)$ then, by Beppo-Levi's Theorem, $I_{n}^{\min }$ converges $($ as $n \rightarrow \infty)$ to its limit

$$
I^{\min }=\frac{1}{\theta_{2}^{2}} \int_{0}^{\infty}\left\{\mu(x)\left(1+\frac{x f^{\prime}(x)}{f(x)}-x \mu(x)\right)^{2}+x^{2} \mu^{2}(x)(\lambda(x)+\mu(x))\right\} d x,
$$

where $\lambda(x)=f(x) /(1-F(x))$ and $\mu(x)=f(x) / F(x)$ is the failure rate and reverse failure rate of $f$, respectively. Obviously, if $I^{\min }<+\infty$, then the Cramér-Rao inequality shows that no consistent sequence of unbiased estimators exists. This, of course, implies that in the corresponding scale family, any sequence of linear (in partial minima) unbiased estimators is inconsistent. The same is clearly true for the location-scale family, because any linear unbiased estimator for $\theta_{2}$ in the locationscale family is also a linear unbiased estimator for $\theta_{2}$ in the corresponding scale family.

In the following we show that there exist distributions with finite variance such that $I^{\mathrm{min}}$ in (6.2) is finite: Define $s=e^{-2}$ and

$$
F(x)=\left\{\begin{array}{cc}
0, & x \leqslant 0, \\
\frac{1}{1-\log (x)}, & 0<x \leqslant s, \\
1-\left(a x^{2}+b x+c\right) e^{-x}, & x \geqslant s,
\end{array}\right.
$$

where

$$
a=\frac{1}{54} \exp \left(e^{-2}\right)\left(18-6 e^{2}+e^{4}\right) \simeq 0.599,
$$




$$
\begin{aligned}
& b=-\frac{2}{27} \exp \left(-2+e^{-2}\right)\left(9-12 e^{2}+2 e^{4}\right) \simeq-0.339 \\
& c=\frac{1}{54} \exp \left(-4+e^{-2}\right)\left(18-42 e^{2}+43 e^{4}\right) \simeq 0.798
\end{aligned}
$$

Noting that $F(s)=1 / 3, F^{\prime}(s)=e^{2} / 9$ and $F^{\prime \prime}(s)=-e^{4} / 27$, it can be easily verified that the corresponding density

$$
f(x)=\left\{\begin{array}{cc}
\frac{1}{x(1-\log (x))^{2}}, & 0<x \leqslant s, \\
\left(a x^{2}+(b-2 a) x+c-b\right) e^{-x}, & x \geqslant s
\end{array}\right.
$$

is strictly positive for $x \in(0, \infty)$, processes finite moments of any order, and has continuous derivative

$$
f^{\prime}(x)=\left\{\begin{array}{cc}
\frac{1+\log (x)}{x^{2}(1-\log (x))^{3}}, & 0<x \leqslant s, \\
-\left(a x^{2}+(b-4 a) x+2 a-2 b+c\right) e^{-x}, & x \geqslant s .
\end{array}\right.
$$

Now the integrand in (6.2), say $S(x)$, can be written as

$$
S(x)=\left\{\begin{array}{cc}
\frac{1-2 \log (x)}{x(-\log (x))(1-\log (x))^{3}}, & 0<x \leqslant s, \\
A(x)+B(x), & x \geqslant s,
\end{array}\right.
$$

where, as $x \rightarrow+\infty, A(x) \sim A x^{4} e^{-x}$ and $B(x) \sim B x^{6} e^{-2 x}$, with $A, B$ being positive constants. Therefore, $\int_{0}^{s} S(x) d x=\int_{2}^{\infty} \frac{1+2 y}{y(1+y)^{3}} d y=\log (3 / 2)-5 / 18 \simeq 0.128$. Also, since $S(x)$ is continuous in $[s,+\infty)$ and $S(x) \sim A x^{4} e^{-x}$ as $x \rightarrow+\infty$, it follows that $\int_{s}^{\infty} S(x) d x<+\infty$ and $I^{\text {min }}$ is finite.

Numerical integration shows that $\int_{s}^{\infty} S(x) d x \simeq 2.77$ and thus, $I^{\min } \simeq 2.9 / \theta_{2}^{2}<$ $3 / \theta_{2}^{2}$. In view of the Cramér-Rao bound this means that, even if a huge sample of partial minima has been recorded, it is impossible to construct an unbiased scale estimator with variance less than $\theta_{2}^{2} / 3$. Also, it should be noted that a similar example can be constructed such that $f^{\prime \prime}(x)$ exists (and is continuous) for all $x>0$.

Of course the above example can be adapted to the partial maxima case by considering the location-scale family generated by the distribution function

$$
F(x)=\left\{\begin{array}{cc}
\left(a x^{2}-b x+c\right) e^{x}, & x \leqslant-s, \\
\frac{-\log (-x)}{1-\log (-x)}, & -s \leqslant x<0, \\
1, & x \geqslant 0
\end{array}\right.
$$

with $s, a, b$ and $c$ as before. 
Acknowledgements. Research partially supported by the University of Athens' Research Fund under Grant 70/4/5637. Thanks are due to an anonymous referee for the very careful reading of the manuscript and also for correcting some mistakes. I would also like to express my thanks to Professors Fred Andrews, Mohammad Raqab, Narayanaswamy Balakrishnan, Barry Arnold and Michael Akritas, for their helpful discussions and comments; also to Professors Erhard Cramer and Claude Lefèvre, for bringing to my attention many interesting references related to log-concave densities. Special thanks are due to Professor Fredos Papangelou for his helpful remarks that led to a more general version of Theorem 4.2, and also to Dr. Antonis Economou, who's question for the Normal Distribution was found to be crucial for the final form of the main result.

\section{References}

[1] Arnold, B.C., Balakrishnan, N. and Nagaraja, H.N. (1992). A First Course in Order Statistics. Wiley, N.Y.

[2] Arnold, B.C., Balakrishnan, N. and Nagaraja, H.N. (1998). Records. Wiley, N.Y.

[3] Bai, Z., Sarkar, S.K. and Wang, W. (1997). Positivity of the best unbiased L-estimator of the scale parameter with complete or selected order statistics from location-scale distribution. Statist. Probab. Lett. 32 181-188.

[4] Bagnoli, M. and Bergstrom, T. (2005). Log-concave probability and its applications. Econom. Theory 26 445-469.

[5] Balakrishnan, N. and Papadatos, N. (2002). The use of spacings in the estimation of a scale parameter. Statist. Probab. Lett. 57 193-204.

[6] Berger, M. and Gulati, S. (2001). Record-breaking data: A parametric comparison of the inverse-sampling and the random-sampling schemes. J. Statist. Comput. Simul. 69 $225-238$.

[7] Burkschat, M. (2009). Multivariate dependence of spacings of generalized order statistics. J. Multivariate Anal. 100 1093-1106.

[8] Chernoff, H., Gastwirth, J.L. and Johns, M.V.Jr. (1967). Asymptotic distributions of linear combinations of functions of order statistics with applications to estimation. Ann. Math. Statist. 38 52-72.

[9] Dasgupta, S. and Sarkar, S.K. (1982). On $\mathrm{TP}_{2}$ and Log-Concavity. In: Inequalities in Statistics and Probability, IMS Lecture Notes-Monograph Series, vol. 5, 54-58.

[10] David, H.A. (1981). Order Statistics. Wiley, N.Y. (2nd. ed.)

[11] David, H.A. and Nagaraja, H.N. (2003). Order Statistics. Wiley, Hoboken (3rd. ed.)

[12] Galambos, J. (1978). The Asymptotic Theory of Extreme Order Statistics. Wiley, N.Y. Belmont, California.

[13] Graybill, F.A. (1969). Introduction to Matrices with Applications in Statistics. Wadsworth, Belmont, California.

[14] Gupta, A.K. (1952). Estimation of the mean and standard deviation of a normal population from a censored sample. Biometrika 39 260-273. 
[15] Hoeffding, W. (1940). Masstabinvariante korrelations-theorie. Scrift. Math. Inst. Univ. Berlin 5 181-233.

[16] Hofmann, G. and Nagaraja, H.N. (2003). Fisher information in record data. Metrika 57 177-193.

[17] Jones, M.C. and Balakrishnan, N. (2002). How are moments and moments of spacings related to distribution functions? J. Statist. Plann. Inference (C.R. Rao 80th birthday felicitation volume, Part I) 103 377-390.

[18] Lehmann, E.L. (1966). Some concepts of dependence. Ann. Math. Statist. 371137 1153.

[19] Lloyd, E.H. (1952). Least-squares estimation of location and scale parameters using order statistics. Biometrika 39 88-95.

[20] Mann, N.R. (1969). Optimum estimators for linear functions of location and scale parameters. Ann. Math. Statist. 40 2149-2155.

[21] Papadatos, N. (2001). Distribution and expectation bounds on order statistics from possibly dependent vatiates. Statist. Probab. Lett. 54 21-31.

[22] Parzen, E. (1979). Nonparametric statistical data modeling. (With comments by John W. Tukey, Roy E. Welsch, William F. Eddy, D. V. Lindley, Michael E. Tarter and Edwin L. Crow, and a rejoinder by the author). J. Amer. Statist. Assoc. 74 105-131.

[23] Prèkopa, A. (1973). On logarithmic concave measures and functions. Act. Sci. Math. (Szeged) 34, 335-343.

[24] Pyke, R. (1965). Spacings (with Discussion). J. Roy Statist. Soc., Ser. B 27 395-449.

[25] Pyke, R. (1980). The asymptotic behavior of spacings under Kakutani's model for interval subdivision. Ann. Probab. 8 157-163.

[26] Resnick, S.I. (1987). Extreme Values, Regular Variation, and Point Processes. Springer-Verlag, N.Y.

[27] Samaniego, F.J. and Whitaker, L.R. (1986). On estimating population characteristics from record-breaking observations I. Parametric results. Naval Res. Logist. Quart. 33 $531-543$.

[28] Samaniego, F.J. and Whitaker, L.R. (1988). On estimating population characteristics from record-breaking observations II. Nonparametric results. Naval Res. Logist. 35 $221-236$.

[29] Sarkadi, K. (1985). On an estimator of the scale parameter. Statist. Decisions Suppl. $2231-236$.

[30] Sengupta, D. and Nanda, A.K. (1999). Log-concave and concave distributions in reliability. Naval Res. Logist. 46 419-433.

[31] Shao, J. (2005). Mathematical Statistics: exercises and solutions. Springer, N.Y.

[32] Smith, R.L.. (1988). Forecasting records by maximum likelihood. J. Amer. Statist. Assoc. 83 331-338.

[33] Stigler, S.M. (1974). Linear functions of order statistics with smooth weight functions. Ann. Statist. 2 676-693. Correction 7, 466.

[34] Tryfos, P. and Blackmore, R. (1985). Forecasting records. J. Amer. Statist. Assoc. 80 $46-50$. 